\title{
Range Finders Using Projected Images
}

\author{
Robert E. Stephens
}

\begin{abstract}
Two optical range finders of six-inch base length have been designed and constructed for making measurements to arbitrary points on a model. One measures distances from 2.5 feet to 10 feet, the other from 9.7 feet to 25 feet. The accuracy of all distances is plus or minus one-quarter inch or better. Because of unusual requirements of use these range finders operate by projecting two images of a bright cross upon the target, which may be fused into a single image by adjustment of the range knob.
\end{abstract}

\section{Introduction}

Two optical range finders of 6 -in. base length have been designed and constructed for making measurements to arbitrary points on a model. One measures distances from 2.5 to $10 \mathrm{ft}$, the other from 9.7 to 25 ft. The accuracy of all distances is $\pm 1 / 4 \mathrm{in}$. or better. Because of the unusual requirements of the application in which they are used these range finders operate by projecting two images of a bright cross upon the target, which may be fused into a single image by adjustment of the range knob.

The problems involved in the choice of the type and base length of the instruments, types of compensators, synchronization of compensation and focusing, adjustment, and calibration are discussed in considerable detail in the following sections.

\section{Base and Magnification}

The ability of a base-type range finder to discriminate between a distance $R_{1}$ and another distance $R_{2}$ depends upon the size of angle $\Delta \alpha$, the difference between the two parallax angles $\alpha_{1}$ and $\alpha_{2}$ subtended by the base of length $B$ at the points $P_{1}$ and $P_{2}$ whose distances are $R_{1}$ and $R_{2}$ (see fig. 1 ). Since $B$ is small compared to $R$, take

$$
\alpha=\frac{B}{R},
$$

and

$$
\Delta \alpha=B \frac{R_{1}-R_{2}}{R_{1} R_{2}}
$$

or, for small differences $\Delta R$,

$$
\Delta \alpha=-\frac{B}{R^{2}} \Delta R .
$$

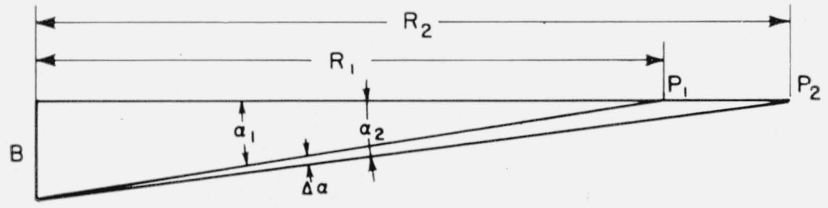

Figure 1. Base $B$, range $R$, and parallax angle $\alpha$ of a basetype range finder.
Range finders are almost invariably telescopic and the angle of disparity presented to the eye of the observer is consequently

$$
\Delta A=M \Delta \alpha=\frac{M B}{R^{2}} \Delta R,
$$

where $M$ is the magnification of the telescope. The negative sign has been dropped because in the following argument only the magnitude is of importance.

The present application requires ability to measure ranges up to $25 \mathrm{ft}$ with an accuracy of $\pm 0.25 \mathrm{in}$. The quantity $\Delta R / R^{2}$ is smallest at the greatest distance; at $25 \mathrm{ft}$ it is $0.25 \div \overline{300}^{2}=2.78 \times 10^{-6}$ radian per inch of base. This is equivalent to $0.573 \mathrm{sec} / \mathrm{in}$.

An average observer can match the two parts of the image in a split-field-type range finder to within approximately $\pm 12 \mathrm{sec}$. His precision of matching for the two parts in the superimposed-image type is a little poorer, about \pm 20 sec. Because of the greater complexity and cost of the split-field type it was decided that the superimposed image would be used if practicable. By eq (2), with $\Delta R / R^{2}=0.573$ sec/in. and $\Delta A=20 \mathrm{sec}$ it is found that $M B=34.9 \mathrm{in}$. We chose to make $M=6$ and $B=6$ in.; this makes $M B=36$ and $\Delta A=20.6 \mathrm{sec}$. At all shorter distances $\triangle A$ will be greater, and the precision of matching well within the required 0.25 in.

\section{Compensators}

A compensator in a range finder is a device that produces a variable angular deviation of the line of sight by way of one window to bring the two images, one by one window and one by the other window, into coincidence. The position of this device when coincidence exists indicates the range. There are several types of compensator each of which has characteristics that make it advantageous for some special application. It is beyond the scope of this report to give a complete discussion of the characteristics of all the types. It will suffice to describe the characteristics necessary and desirable for our specific application and to show that the chosen type has these, or most of them.

The first characteristic to be considered is the sensitivity. This may be defined as the increment of 
displacement of the compensator corresponding to the smallest increment of range that is to be measurable. The sensitivity is not constant but increases from a minimum at the long-range end of the scale to a maximum at the short-range end. This feature definitely limits the spread between the maximum and minimum distances measurable by one range finder. Adequate sensitivity must be provided at the long-range end of the scale; at shorter ranges the sensitivity increases until such a large movement is necessary for a small change in distance that making a match becomes intolerably slow.

The second consideration in the choice of the compensator is the law relating the displacement of the compensator to the resulting deviation of the line of sight. For our purpose a compensator whose deviation angle is proportional to the displacement is desirable. Important reasons for this are that adjustment and calibration are simplified, and that it facilitates coupling to a focusing mechanism, which will be discussed in detail later.

Another consideration is simplicity of construction. If the other conditions are satisfied the compensator of the simplest construction is the obvious choice.

The sliding-lens compensator seemed to fit these requirements better than any other type. This consists of a low-power lens that is larger than the window and may be moved across the window along a line parallel to the base. A schematic drawing of this is shown in figure 2. In (a) the lens is shown centered on the line of sight, giving no deviation. In (b) the lens is displaced so that the line of sight goes through it eccentrically and suffers a deviation $D$. The magnitude of the deviation is proportional to the eccentricity (this is an approximation of sufficient accuracy for the purpose). Let $D$ be the deviation, $x$ the eccentricity of the lens, and $\Phi$ the power of the lens, then

and

$$
D=x \Phi,
$$

$$
\Delta D=\Phi \Delta x
$$

In section 2 it was determined that the change in parallax angle represented by $0.25 \mathrm{in}$. at a distance
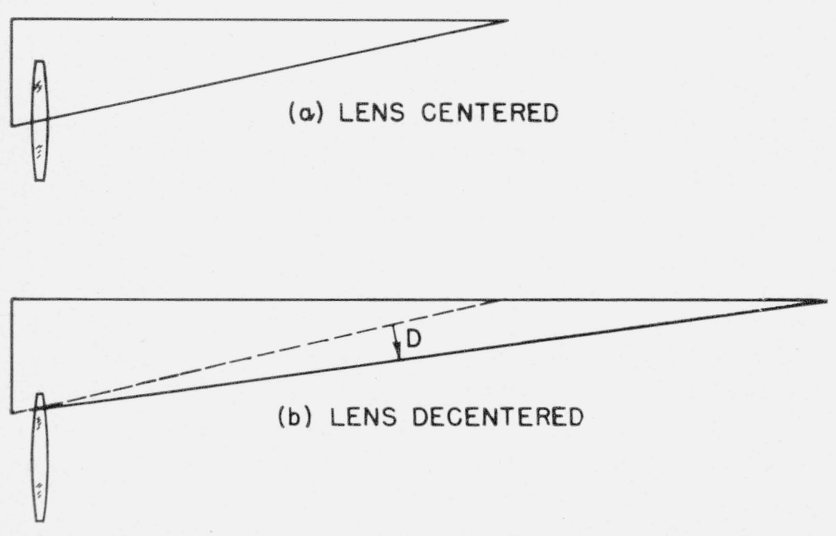

Figure 2. The action of a sliding-lens compensator. of $25 \mathrm{ft}$ was $2.78 \times 10^{-6}$ radian per in. of base length. Also it was decided that a base length of 6 in. would be used. The minimum increment in parallax angle that is to be perceived and measured is then $16.67 \times 10^{-6}$ radian. It is convenient to first compute the eccentricity of a 1-diopter lens for a deviation of $16.67 \times 10^{-6}$ radian. This is, of course, $16.67 \times 10^{-6} \mathrm{~m}$ or $0.01667 \mathrm{~mm}$. This can be measured with sufficient accuracy by a micrometer screw with spring preloading to take up the play. A 1-diopter lens is therefore the choice for the compensator.

For the experimental model it would be impracticable to rule a special scale indicating distance directly, and it was decided to use a commercially available linear scale indicating in hundredths of a turn up to ten turns. Consequently it was decided that the compensator would be decentered by a precision screw whose position would be indicated by one of these dials. This makes the scale 1,000 divisions long. These divisions are of such a size and quality that estimation to one-half division is easy and of sufficient accuracy. It was therefore decided to let one scale division represent approximately 0.5 in. at a distance of $25 \mathrm{ft}$.

We have computed the decentration corresponding to $0.25 \mathrm{in}$. at $25 \mathrm{ft}$ to be $0.01667 \mathrm{~mm}$. Our entire scale is to be 2,000 times this long, approximately; this is $33.33 \mathrm{~mm}$. The desired lead for the screw then is one-tenth of this or $3.333 \mathrm{~mm}$ per turn. One-eighth in. is $3.175 \mathrm{~mm}$, and because screws of this lead can be made on easily available machines this was adopted for the construction of the instrument. The total available range of displacement for the compensator is $1.25 \mathrm{in}$. or $31.75 \mathrm{~mm}$. with this screw. The total change of deviation is then, by eq (5), 0.03175 radian. At $25 \mathrm{ft}$ the parallax is 0.02 radian. If the instrument is built with a bias of approximately 0.035 radian it can be made to work from a parallax angle of 0.02 to 0.05175 radian. This latter parallax corresponds to a distance of $9.66 \mathrm{ft}$, where one division of the scale represents $0.071 \mathrm{in}$. The total distance range of the instrument then is from 9.66 to $25 \mathrm{ft}$. It would be rather impracticable to try to increase this total range by increasing the length of the scale because, at the short-distance end of the scale the instrument is already much too sensitive. Even if the length of the scale were doubled it would only extend the range down to $6.2 \mathrm{ft}$. It is therefore obvious that another instrument with a less sensitive compensator is necessary for distances shorter than about $10 \mathrm{ft}$.

To measure distances under $10 \mathrm{ft}$, a sliding lens compensator of sufficient power and low sensitivity for a range finder of 6 -in. base would have to be corrected for spherical and chromatic aberrations, and coma. This would make it so complex that another type of compensator was chosen for the second instrument. A rotating mirror has the desired characteristics - it has no aberrations regardless of the magnitude of the deviation produced; and its sensitivity is inherently low because the angle of rotation of the mirror is only half the resulting deviation. The 
sensitivity can be made adequate by the use of a long lever arm for producing the rotation of the mirror.

At a distance of $10 \mathrm{ft}$ the parallax angle for a 6 -in. base is 0.050 radian. For an increment of distance of 0.25 in. the change in parallax is 0.0001042 radian. If as before we let this be the change corresponding to one-half scale division the entire scale of 1,000 divisions will cover a range of 0.2084 radian. The minimum distance will then correspond to $0.05+$ 0.2084 , or 0.2584 radian. This distance is $1.935 \mathrm{ft}$, or 23.2 in.

The increment of parallax corresponding to 0.25 in. at a distance of $10 \mathrm{ft}$ is 0.0001042 radian or 21.5 sec. This is large enough for satisfactory matching to within \pm 0.25 in. without magnification. At any shorter distance it is still larger and thus more than adequate for matching with the required precision without telescopic magnification.

\section{Projection}

These range finders are to be used for measuring the slant distance to a particular point on a scale model. Because various points on the model are to be chosen for observation at various times the models are painted a uniform white and there is no permanent mark whose two images could be bought to coincidence in a range finder. It is necessary to provide a temporary mark and desirable that this be done without disfiguring the model. This can be done by working in a darkened room and projecting a tiny spot of light on the desired part of the model.

If an optical range finder of the usual type were used the location of the projector would be unimportant, so long as a small bright spot could be projected to the proper place on the model. The observer would look into the eyepiece of the range finder, turn the knob to achieve coincidence, then obtain the distance by reading the scale. Because of the diverse locations of the model the observer would have to be prepared to follow the eyepiece, and take whatever position would be necessary to sight in the direction of the model. Complex construction might be resorted to, to bring the image from any direction into a fixed eyepiece. However, in this application such construction was impractical, partly because of the space it would require and partly because of rather considerable extra cost.

An alternative is to construct the range finder so that it projects two bright images of a cross, one through one window and another through the other window, that are to be brought into coincidence at the desired point on the model by means of the compensator. A telescope of suitable magnifving power, located in any convenient place, could be used for viewing the images while adjusting them into coincidence. This arrangement, although still requiring some variation in the position of the observer and direction of view, allows viewing without impracticable contortions. This is the plan that was adopted and the range finders accordingly were designed to contain the means for projecting two bright images upon the model.

\section{Focusing}

In order that coincidence may be established with sufficient precision to assure the requisite precision of the distance measurement, the projected images must be focused sharply on the model at the point to which the distance is to be measured. Adjustment of the focus and adjustment of parallax to achieve coincidence could very well be independent. However, this would necessitate two separate adjustments for each measurement and would consequently be somewhat inconvenient and neces.sarily slower. Fortunately the laws governing the motion of the compensator and the focusing of a lens are so similar that it is easy to provide mechanical coupling of the two functions to achieve simultaneously both coincidence and sharp focus.

Both the sliding lens and the rotating mirror produce angular deviations of the line of sight that are proportional to the displacement of the compensator, thus:

$$
D=K x
$$

where $D$ is the deviation, $x$ the displacement of the compensator (a length for the sliding lens and an angle for the rotating mirror), and $K$ is a constant. For the purpose of this discussion the displacement of the compensator, $x$, is measured from the position it would have to make the parallax angle, $\alpha$, of the two lines of sight equal to zero, that is, when the two lines of sight are parallel. Then $D$ of eq (6) equals $\alpha$ of eq (a) and consequently

$$
x=\frac{B}{K R} .
$$

The law relating the object distance $l$ and the image distance $l^{\prime}$ for image formation by a lens is expressible in several forms. The one that is convenient for this discussion is the Newtonian form,

$$
l l^{\prime}=f^{2},
$$

where $l$ and $l^{\prime}$ are the object and image distances measured from their respective principal foci, and $f$ is the equivalent focal length of the lens. If the range finder and projection lens were so positioned that the front focal point of the lens coincided with the point from which the distance was measured (a condition that exists nearly enough in practice), $R$ and $l^{\prime}$ would be equal. Since $R=B / K x, l^{\prime}=f^{2} / l$, and $B / K x=f^{2} / l$,

$$
\text { thus } \quad l=\frac{K f^{2}}{B} x \text {. }
$$

Since $K, f^{2}$, and $B$ are constants, $l$ is proportional to $x$. Consequently the two movements may be coupled by a simple mechanical device such as a lever or gears having the ratio $K f^{2} / B$.

\section{Design}

The superimposed-image type of range finder makes use of what is known as a "beam splitter," 


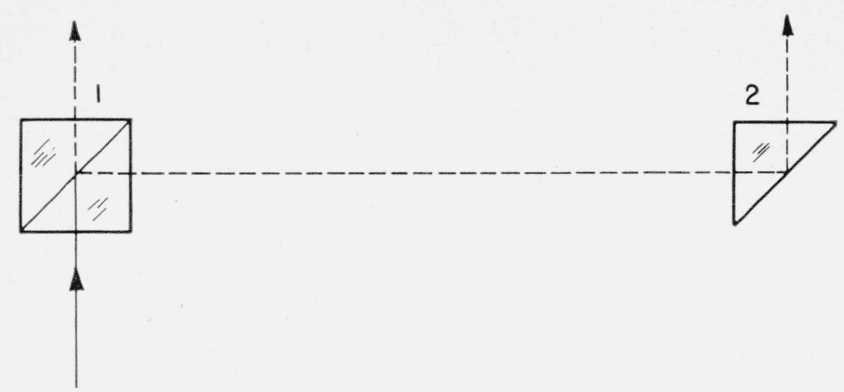

FIGURE 3. The elements of a superimposed-image range finder.

a surface made either of a very thin layer of metal or a multiple layer system of dielectric films. This is so made that at the desired angle of incidence the reflectance is approximately equal to the transmittance. The schematic diagram of a range finder of this type is shown in figure 3 . The beam splitter is in the cube at 1 , which is made by fastening together with optical cement the equal hypotenuse faces of two right angle prisms, one of which has the beam-splitting layer on it. The resulting cube is a beam splitter that has the same length of glass in both paths, transmitted and reflected. Thus light entering along the solid line of figure 3 is split into two parts approximately at right angles to one another and, except for a minor difference in intensity, these are both alike.

The optical system of the long-range instrument is shown in figure 4 . The reticle, $r$, is illuminated by lamp, $l$, through condensing lens, $c$. The projection lens, $L_{3}$, projects an image of $r$ to the appropriate distance. The projected beam passes through the beam-splitting cube behind window 1 where it is split into two parts of approximately equal intensity, one transmitted and emerging at 1 , the other reflected and finally emerging at 2 . The position of the beam splitter is such that the two parts of the beam emerge from it approximately at right angles. The pentaprism, $P$, is used to fold the projector beam so as to reduce the bulk of the instrument.

The large, low-powered lens, $L_{1}$, is the compensator. It is movable along a line approximately parallel to the base line of the instrument. The fixed concave lens, $L_{2}$, has diverging power equal to the converging power of the compensator; its function is to nullify the converging effect of the compensator. The two lenses together form, in effect, a prism of variable angle with no power of convergence or divergence.

The right-angled prism behind window 2 is oriented so that in the absence of deviation by the compensator the beam emerging from window 2 intersects that from window 1 at a distance whose parallax angle is the mean of those for the maximum and minimum ranges of the instrument. This bias allows the compensator lens to function over a range that is centered on the lens thus minimizing the effects of aberrations and permitting a simple uncorrected lens to be used.

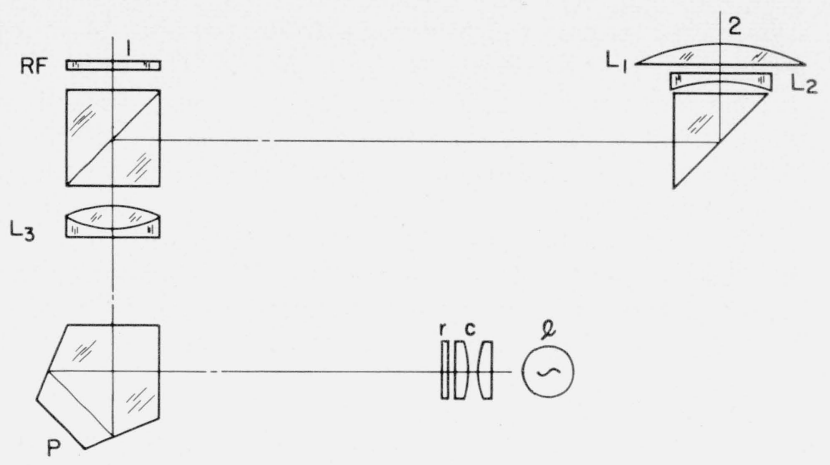

FiguRE 4. Schematic diagram of long-range instrument.

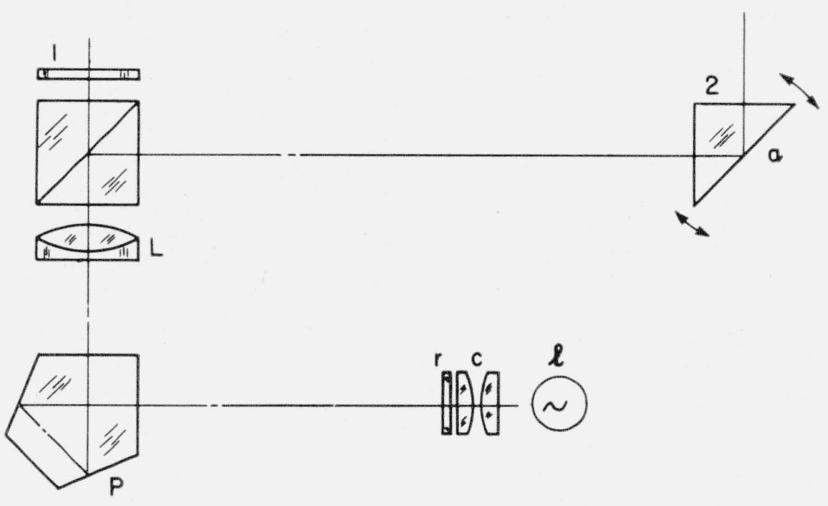

FiguRE 5. Schematic diagram of short-range instrument.

The direction-determining image, from window 1 , is colored red by a filter designated $R F$. It was originally intended that the other image would be colored blue because of the improved precision of setting in coincidence that is usually achieved when the images are in complementary colors. However, the inclusion of a blue filter so reduced the brightness of the image that the hue became almost inappreciable. The precision of setting was reduced rather than increased because of the low brightness. The blue filter was consequently omitted.

The assembly consisting of reticle, condensing lens, and lamp is mechanically linked to the compensator so that the image of the reticle is focused at the same distance at which the two beams emerging from 1 and 2 intersect.

The short-range instrument is shown schematically in figure 5; it differs only in the compensator. The right-angled prism at 2 in this case instead of being fixed in position is mounted so that it may be rotated through several degrees on an axis normal to the plane of the diagram at point $a$. The beam from the window at 2 may thus be varied in direction so as to intersect the other beam at distances from approximately 2 to $10 \mathrm{ft}$. The reticle-condenser-lamp assembly is linked to the compensator prism so that as the prism is rotated this assembly is displaced along the line of projection thus maintaining the focus at the same distance at which the beams from 1 and 2 intersect. 
In the long-range instrument the movement of the compensator is several times that of the reticle. To minimize the effect of mechanical play between the scale and the compensator and to make the mechanical advantage favorable, the precision screw, to which the scale is attached, drives the compensator directly. In the short-range instrument the movement of the reticle is large compared to the displacement of the lever that rotates the prism compensator. In this case the screw drives the reticle assembly directly. The mechanical play is reduced to inconsequential magnitude by preloading at all points of contact in the linkage.

The beam of light projected through window 2 will travel farther than that through window 1 in reaching the point where they intersect and the images are supposed to be coincident. Because of this the two images cannot both be in sharp focus. The best compromise is to have them focused so that the sharp focus of the beam from 1 is beyond the point of intersection and that from 2 is ahead of it. Then both will be sufficiently sharp for the purpose. Since the two images will be the same size only at their respective positions of sharp focus, one will be smaller and the other larger at their position of coincidence. This reduces the precision of setting but not enough to be serious in this application.

The positions of the points, $F_{1}$ and $F_{2}$, from which $l_{1}^{\prime}$ and $l_{2}^{\prime}$, the two image distances, are measured are illustrated in figure 6 . Here $F_{1}$ is the front principal focus of the projection lens, $L$, for the transmitted beam emerging from window 1 , and $F_{2}$ is the front principal focus for the beam reflected by the beam splitter and emerging from window 2 . These are the points from which $l_{1}^{\prime}$ and $l_{2}^{\prime}$, respectively, are measured. However, the distance $R$ to the observed point is measured from the base line.

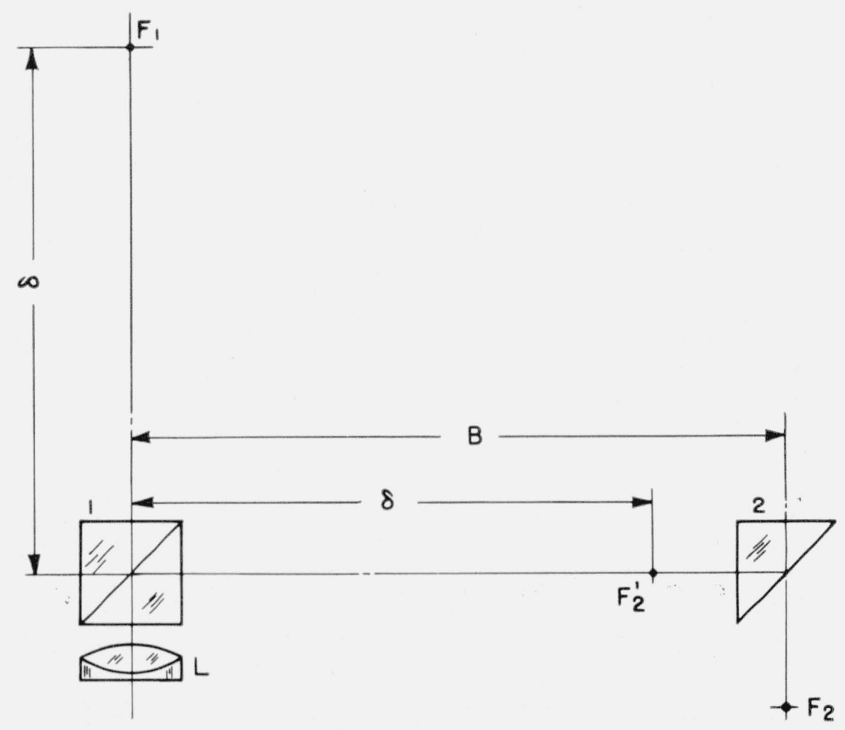

Figure 6. Positions of front focal points for beams transmitted and reflected at beam splitter.
If the images were focused so that $l_{1}^{\prime}=l_{2}^{\prime}=R$, then the point of coincidence would not be exactly half way between the two sharp images, because in our range finders $\delta=110 \mathrm{~mm}$, approximately, and the base length is $150 \mathrm{~mm}$. The coincidence point would be much closer to the image from window 2, and also the distances of the sharp images from the point of coincidence would remain constant at all ranges if the motion of the reticle were coupled to that of the compensator with the ratio $k f^{2} / B$, in accordance with eq 9. Although this condition is not optimum it might be satisfactory because of the depth of focus.

It is possible, however, to maintain a more nearly optimum condition. If the ratio of the linkage is made slightly different from that of eq 9, and the reticle position changed slightly, it is possible for two different ranges to locate the sharp images equidistant from the point of coincidence. If these ranges are chosen properly, somewhere near but not at the ends of the scale, the condition of focus will change only slightly from one end of the scale to the other.

For the short-range instrument the linkage ratio was computed so that the two images would be equidistant from the point of coincidence when the distance of coincidence was $1.0 \mathrm{~m}$ and $2.7 \mathrm{~m}$. At these distances $l_{1}=R+.075-0.110$ or $0.965 \mathrm{~m}$ and $2.665 \mathrm{~m}$. The conjugate values of $l$ are 0.018056 and $0.006538 \mathrm{~m}$ for $f=0.132 \mathrm{~m}$ ). The difference, $\Delta l=0.011518$. The corresponding values of the parallax angle are 0.15000 and 0.05556 radian; $\Delta \alpha=0.094444$. Consequently $\Delta l / \Delta \alpha=0.1219 \mathrm{~m}$ per radian. Because the rotation of the mirror-type compensator is only half the resulting change in the parallax angle the linkage ratio is $2 \times \Delta l / \Delta \alpha=0.2438 \mathrm{~m}$ per radian. This, converted to more appropriate units, is $4.255 \mathrm{~mm}$ per degree. For comparison the value of $K f^{2} / B$ is $4.055 \mathrm{~mm}$ per degree. For other ranges the distances from the point of coincidence to the sharp images are not equal, but the discrepancy is small. It is shown graphically in figure 7 . The two ranges of optimum focus were chosen as a result of a previous trial.

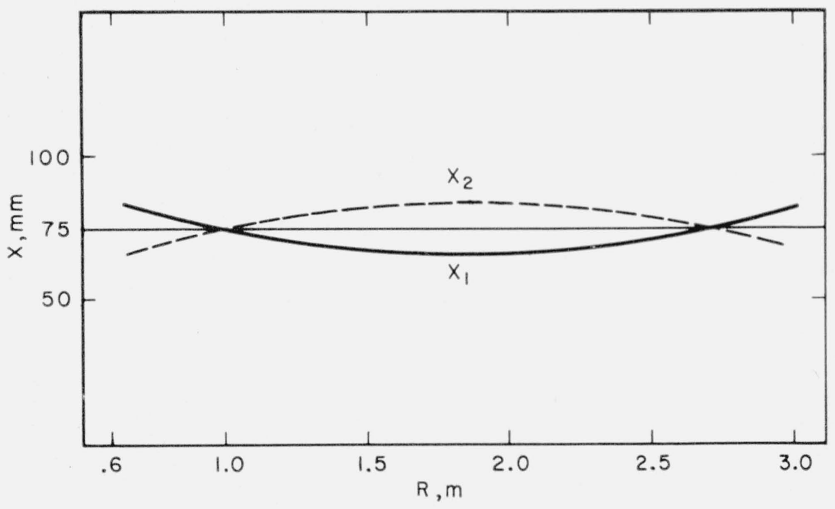

FIGURE 7. The distances, $x_{1}$ and $x_{2}$, from the point of coincidence at distance $R$ to the sharp images of the reticle projected through windows 1 and 2 (both plotted as positive). 
Similar analysis for the long-range instrument gives an optimum linkage ratio of 0.118 . This is dimensionless and means that the displacement of the reticle is 0.118 times the displacement of the sliding-lens compensator. In this case the tracking errors are completely negligible.

It is not possible to realize this accuracy of synchronization by construction in which practicable optical and mechanical tolerances are allowed. Consequently the linkages were made adjustable, and the correct ratio achieved by experiment.

\section{Telescope}

A telescope is necessary for \pm 0.25 -in. accuracy with the long-range instrument. A 90-degree elbow type was adopted so that the position of the eyepiece and direction of viewing would permit comfortable use regardless of the direction to the object. It is constructed of components from a $6 \times 30$ binocular except that a pentaprism has been substituted for one of the customary Porro prisms to give the 90degree bend to the line of sight. Also the spacings of the components have been changed slightly so that the range of focusing encompasses the working range of the range finder, 9.7 to $25 \mathrm{ft}$. The optical layout of the telescope is shown in figure 8 .

\section{Adjustment}

No attempt will be made to describe in detail the tedious procedures found necessary to adjust the instruments as received from the shop. An outline of these adjustments and the means for making them will suffice. The necessity for adjustments arises from the fact that the requisite accuracy of the finished instrument can not be achieved by the application of tolerances to the separate parts. The following characteristics must be adjusted after assembly:
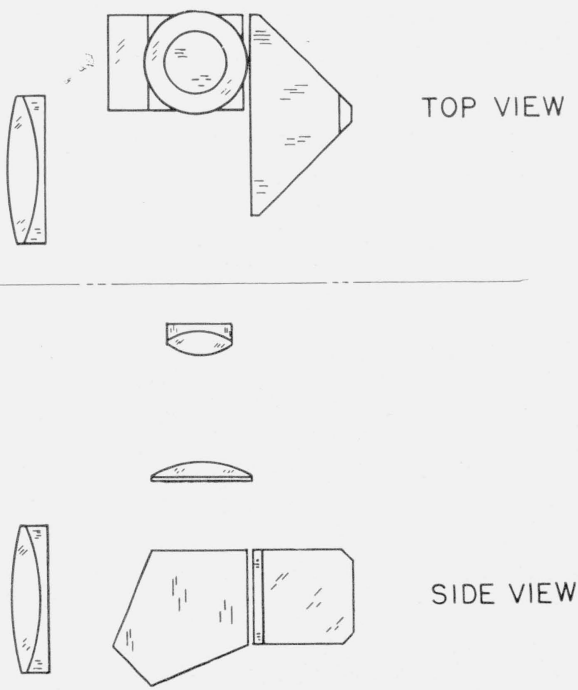

Figure 8. Elbow telescope.

\subsection{The Bias}

This is the adjustment that makes the range between zero and maximum displacement of the compensator correspond to the desired maximum and minimum ranges for the instrument. The bias of either instrument is adjusted by slight turning of the right-angle prism around an axis parallel to its axis. Simultaneous with this adjustment any existing dip-vergence ${ }^{1}$ is corrected by the use of shims to tilt this prism. When the prism has been correctly placed it is clamped, and locating retainers are screwed down so as to hold it permanently in this position.

\subsection{Focus}

The focus is adjusted for maximum sharpness of both images by sliding the reticle longitudinally in its carriage. Because of the construction the transverse adjustment of the reticle is likely to be disturbed by the focusing adjustment. Because of this the alinement of axes is left until last.

\subsection{Focusing Linkage Ratio}

This must be adjusted to achieve optimum tracking between focusing and compensation to produce simultaneous coincidence and focus over the entire distance range of the instrument. The ratio of the levers between the compensator and the reticle carriage is adjusted so that at two positions, approximately one-eighth of the total distance range from each end of the range, the two sharp images are equidistant from the position of coincidence. Provision for adjusting either the length of one arm or the position of the fulcrum has been made in the construction of the instruments. The focusing will require adjustment again after each change in the lever. Once the correct ratio and focus have been obtained at these two distances the departure from optimum focus at other distances will be negligible.

\subsection{Pointing Accuracy}

The image from window 1 , or both images when in coincidence, is used for determining the azimuth and elevation of the line of sight. The direction of the line of sight, therefore, must not change with change in focus.

\subsection{Alinement of Optical and Mechanical Axes}

One of the three axes of the mounting approximately coincides with the base line of the range finder. When the circular scale that indicates rotation around this axis is set at zero, the line of sight must coincide with one of the other two mechanical axes.

Adjustment to aline the optical axis with the mechanical one and to allow focusing without changing the direction of the optical axis is a tedious one. It is accomplished by achieving simultaneously the

1 The projected beam from window 2 must, of course, intersect that from window 1 at any setting of the compensator. If it does not it is not possible to bring the images into coincidence. Such disparity is called dip-vergence. 
proper position and tilt of the pentaprism and proper transverse position of the reticle.

\section{Calibration}

The parallax angle, $\alpha$, of a range finder is given by eq (1). This angle is a linear function of the displacement of the compensator, thus

$$
\alpha=K s+\alpha_{o},
$$

where $s$ is scale reading and $\alpha_{o}$ is a constant. This, substituted into eq (1), gives

$$
K s+\alpha_{o}=\frac{R}{R} .
$$

However, none of the constants $K, \alpha_{0}$, or $B$ is known precisely; consequently the relation between the distance of coincidence and the scale reading must be determined experimentally. It is neither necessary nor desirable to evaluate these constants explicitly.

If eq (11) is divided by $B$, the result is

$$
\frac{K}{B} s+\frac{\alpha_{o}}{B}=\frac{1}{R}
$$

or, since $K / B$ and $\alpha_{o} / B$ are instrumental constants, let us rewrite the relation as

$$
P s+Q=\frac{1}{R} .
$$

Theoretically $P$ and $Q$ could be evaluated from the data obtained by setting for coincidence and reading the two scale readings corresponding to any two known distances. However, the accuracy of that method is insufficient. Settings should be marje for perhaps tive different known distances, the average of the scale reading for ten successive settings being used as the value of $s$ for each distance. The constants may then be evaluated by the leastsquares method or the method of averages. A method that should give sufficient accuracy for the purpose is to plot points on a large-scale graph corresponding to each of the known distances using as abscissae the values of $s$ and as ordinates the values of $1 / R$. It is then a matter of judgment to draw the straight line that best fits the data. The constants may then be evaluated from two widely spaced arbitrary points on the line. The line itself may be used as the calibration but is inconvenient because it is plotted with respect to the reciprocal of $R$ instead of $R$ directly. It is more convenient to have a table giving $R$ as a function of $s$. This may be computed from eq (12) once accurate values of the constants have been obtained.

In subsequent use of the instrument an occasional check of the correctness of the scale should be made by making several settings at a known distance. If there is any discrepancy it can be corrected by repositioning the pointer. This procedure is theoretically exact because of the nature of the law governing the compensator.

Washington, August 22, 1957. 